\title{
The targeting ability of fluorescent quantum dots to the folate receptor rich tumors
}

\author{
Tristan Mangeolle $\mathrm{a}^{\mathrm{a} b}$, Ilya Yakavets ${ }^{\mathrm{a}, \mathrm{b}, \mathrm{c}}$, Nicolas Lequeux ${ }^{\mathrm{d}}$, Thomas Pons ${ }^{\mathrm{d}}$, Lina Bezdetnaya ${ }^{\mathrm{a}, \mathrm{b}, *}$, \\ Frédéric Marchal ${ }^{\mathrm{a}, \mathrm{e}}$ \\ ${ }^{a}$ Centre de Recherche en Automatique de Nancy, Centre National de la Recherche Scientifique UMR 7039, Université de Lorraine, Campus Sciences, Boulevard des \\ Aiguillette, 54506 Vandoeuvre-lès-Nancy, France \\ ${ }^{\mathrm{b}}$ Research Department, Institut de Cancérologie de Lorraine, 6 avenue de Bourgogne, 54519 Vandoeuvre-lès-Nancy, France \\ ${ }^{\mathrm{c}}$ Laboratory of Biophysics and Biotechnology, Belarusian State University, 4 Nezavisimosti Avenue, 220030 Minsk, Belarus \\ ${ }^{\mathrm{d}}$ LPEM, ESPCI Paris, PSL Research University, CNRS UMR 8213, Sorbonne Université, 10, rue Vauquelin, 75005 Paris, France \\ ${ }^{\text {e }}$ Surgical Department, Institut de Cancérologie de Lorraine, 6 avenue de Bourgogne, 54519 Vandoeuvre-lès-Nancy, France
}

\section{A R T I C L E I N F O}

\section{Keywords:}

Photodiagnosis

Nanoparticles

Quantum dot

Folic acid

Multicellular tumor spheroids

\begin{abstract}
A B S T R A C T
Background: Quantum dots (QDs) bring new insights in cancer theranostics. Exceptional brightness together with the simple possibility to modify surface with targeting molecules make QDs attractive agents in fluorescence guided surgery and photodynamic therapy. Currently, many targeted QDs have been developed for theranostic purpose. However, their targeting ability was tested mainly in two dimensional monolayer tumor cell models, while our study includes 3D tumor model reflecting the specificity of in vivo tumor environment.

Methods: Core/multilayer shell CdSe/CdS/ZnS QDs were conjugated with folic acid (FA) and characterized spectroscopically. Cytotoxicity of QDs on KB and A549 cells lines were evaluated using the MTT assay. Cellular uptake of QDs was assessed by epifluorescent microscopy. To study the distribution of QDs in tumor tissue, KB spheroids were prepared by means of the liquid overlay technique and then frozen cut of spheroids treated with QDs were imaged by epifluorescence microscopy.

Results: We confirmed the specificity of QD-FA for the folic acid receptor positive KB cells. In 3D tumor spheroid model we demonstrated uptake enhancement of QD-FA compared with non-targeted QD. It was demonstrated that penetration profiles were similar for both QDs with penetration depth never exceeding $100 \mu \mathrm{m}$.

Conclusions: We have demonstrated the effectiveness of FA conjugated QDs to target tumor spheroids thus confirming the crucial role of FR $\alpha$ receptor as a target. Further improvement of QD-FA targeting ability could be performed using dual targeting different targeting agents, such as FA and cyclic RGD.
\end{abstract}

\section{Introduction}

Cancer is the second most important cause of death worldwide [1]. Current therapeutic improvements are mainly related to tumor targeting with nanoparticles (NPs), which show superior properties compared with routine drugs. NPs display improved tumor accumulation through both passive and active targeting, large surface to volume ratio and versatile surface chemistry. Moreover, they can associate therapeutics and imaging capabilities of drugs with theranostic aims [2]. Among various types of inorganic NPs, quantum dots (QDs) have undoubtedly affected bioimaging and drug delivery research, more than any other nanomaterial [3]. QDs are fluorescent nanoparticles with exceptional brightness, strong photostability, and adaptable surface chemistry. A great deal of current research and clinical trials are devoted to the assessment of the potential use of QDs as an alternative fluorescent marker [4-8]. Moreover, by using QDs fluorescent probe for fluorescence guided surgery and as a drug nanoplatform, QDs can achieve theranostic purpose [9,10].

Poor water-solubility and disappointing biocompatibility of highquality hydrophobic QDs limited their biological applications. It has been reported that water transfer with amphiphilic polymer (e.g. with poly(ethylene-glycol)) encapsulation and ligand exchange provides promising potential to overcome the hydrophobicity barrier and to improve the biological properties of bare uncoated QDs. At the same

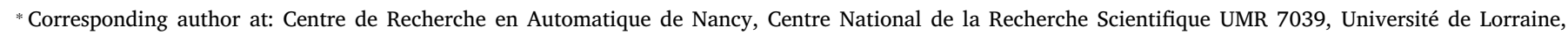
Campus Sciences, Boulevard des Aiguillette, 54506 Vandoeuvre-lès-Nancy, France.

E-mail addresses: tristanmangeolle1990@hotmail.fr (T. Mangeolle), i.yakavets@nancy.unicancer.fr (I. Yakavets), nicolas.lequeux@espci.fr (N. Lequeux), thomas.pons@espci.fr (T. Pons), 1.bolotine@nancy.unicancer.fr (L. Bezdetnaya), f.marchal@nancy.unicancer.fr (F. Marchal). 
time, the polymer coating limits the accumulation and selectivity of NPs [11]. Active targeting could be a solution to overcome the lack of QD selectivity. Efficient tumor targeting by QDs can be achieved using tumor-specific ligands such as antibodies, peptides, sugars of vitamins. In the present work, we used QDs coated by a zwitterionic copolymer ligand to assess the improvement of QD selectivity upon their conjugation with folic acid (FA) as a targeting agent.

To the best of our knowledge, FA has attracted wide attention as a targeting agent for tumor detection and drug delivery [12,13]. FA is a highly stable, is compatible with both organic and aqueous solvent, it possesses non-immunogenic character, low molecular weight and can conjugate a wide variety of molecules [14]. Moreover, the folate receptor- $\alpha$ (FR- $\alpha$ ) is a well-known cancer cell associated protein which can actively internalize folate bound NPs via endocytosis [13]. FR- $\alpha$ is overexpressed in many solid malignancies, such as breast, ovary and lung cancer [15-17].

Many drugs, nucleic acid, paramagnetic or fluorescent imaging agents have been associated with folate, few of them are currently undergoing clinicals trials [18]. Moreover, many folate conjugated QDs (QD-FA) were developed for therapeutics purpose [19-22]. As an example, QD-FA was even proposed as a potential photosensitizer in photodynamic therapy of cancer [23].

It is worth noting that previous studies were mainly focused on synthesis and active targeting of QD-FA on cell monolayer models, which do not reflect accurately the microenvironment of in vivo tumors [24]. The novelty of the present study is the use of three dimensional (3D) multicellular tumor spheroids (MCTSs), which resemble small avascular tumors [25], thus providing better insight into targeting ability and intratumor penetration of nanomedicines. In the present work, we developed the QDs conjugated to FA and assessed their targeting ability in MCTSs made from FR- $\alpha$-positive oral cancer cells (KB). In the parallel we evaluated QDs uptake in conventional monolayer cells model.

\section{Materials and methods}

\subsection{Quantum dots synthesis, coating and characterization}

Core/multilayer shell CdSe/CdS/ZnS QDs $(6-7 \mathrm{~nm})$ were synthetized as described elsewhere [26,27]. Briefly, core was synthetized through solvothermal process with cadmium myristate and selenium powder as precursor in oleic acid. A multilayer shell was grown through successive ion layer adhesion and reaction (SILAR). The structure of the QDs and of the ligand used for anchoring folic acid is depicted in the

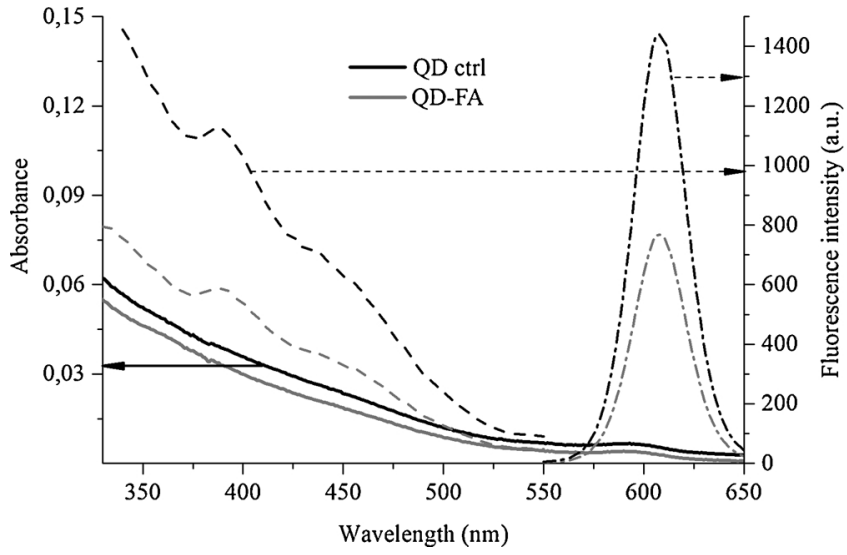

Fig. 1. Spectra of absorbance (solid line), fluorescence excitation (dashed line, $\lambda_{\mathrm{em}}=608 \mathrm{~nm}$ ) and fluorescence emission (dashed-dotted line, $\lambda_{\text {exc }}=340 \mathrm{~nm}$ ) of control QDs (QD-ctrl) (black line) and QDs conjugated to FA (QD-FA) (red line) in PBS. The concentration of QDs was $10 \mathrm{nM}$.

\section{Scheme 1.}

To transfer hydrophobic QDs in aqueous media, a two-step ligands exchange was used. First, QDs were coated with 3-mercaptopropionic acid (MPA), a short labile ligand, to transfer them into water. Then, they were exchanged with a block copolymer ligand composed of a first block of poly(vinylimidazole) that bind to the QD surface, and a second statistical block composed of a sulfobetaine-based monomer and a primary-amine containing monomer in a 7:1 ratio [28]. After purification by ultracentrifugation and ultrafiltration, the primary amines on the QD ligands (1 molar equivalent, corresponding typically to $2.5 \mathrm{nmol}$ of QDs) were reacted successively with 600 eq. bis(sulfosuccinimidyl) suberate for $1 \mathrm{~h}$ at $\mathrm{pH} 7$, then 1000 eq. of $\mathrm{PEG}_{2000}$-bis (NHS) for $4 \mathrm{~h}$ at $\mathrm{pH} 7$, then with (QD-FA) or without (QD-Ctrl) $200 \mathrm{eq}$. folate-NHS for $1 \mathrm{~h}$ at $\mathrm{pH}$. The remaining primary amines were then finally reacted with 1000 eq. of bis(sulfosuccinimidyl) suberate for $1 \mathrm{~h}$ at $\mathrm{pH}$ 7. Between each step, excess reactants were removed by three steps of ultrafiltration (Vivaspin-500, $100 \mathrm{kDa}$ cutoff). QD concentrations were determined from the absorbance of the core CdSe QD solution, as detailed in [29].

UV-vis spectroscopy measurements were conducted using Lambda 35 spectrophotometer (Perkin Elmer, Waltham, USA) in the wavelength range $325-650 \mathrm{~nm}$ in transmission mode using $1 \mathrm{~cm}$ quartz cuvette. Emission and excitation spectra were acquired using LS55

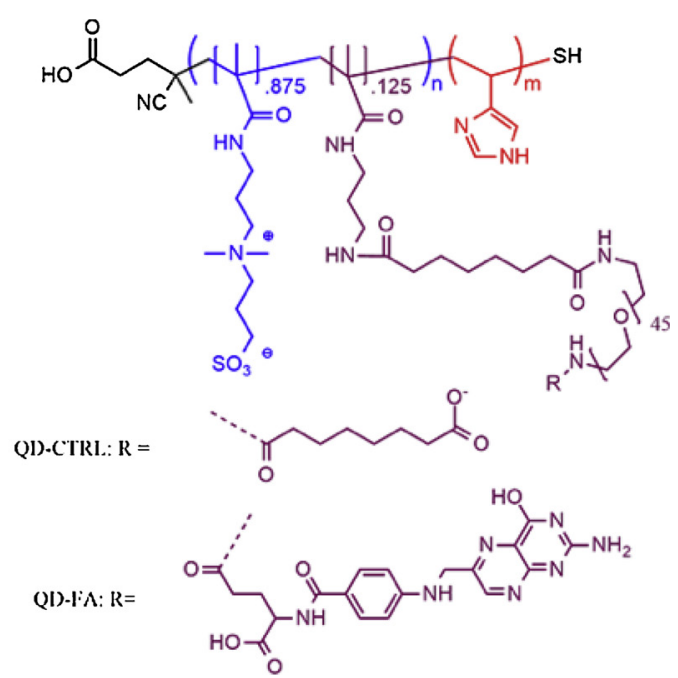

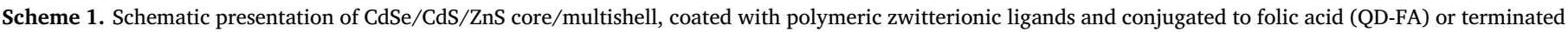
by a terminal carboxylate group (control QD). 


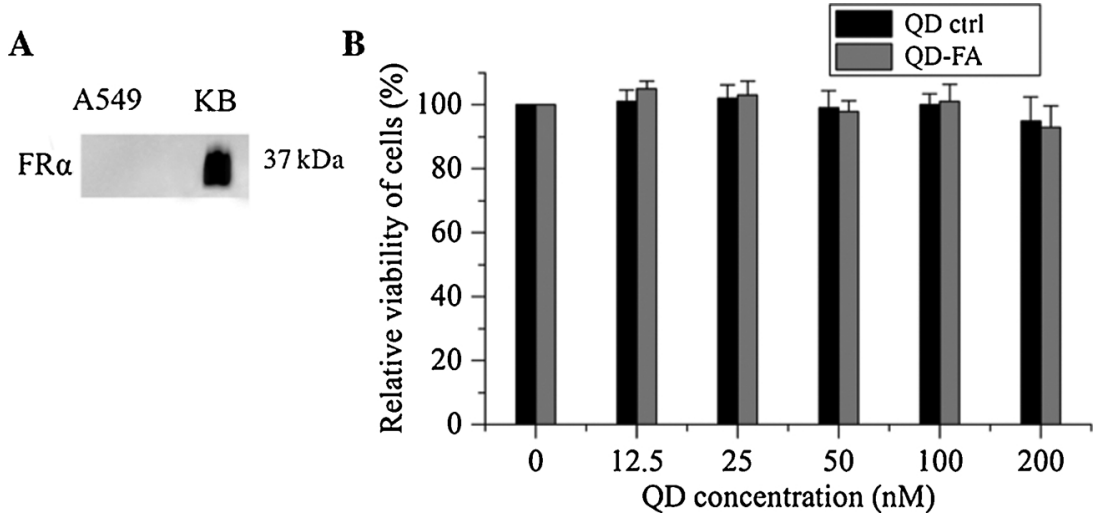

Fig. 2. (A) Protein expression by Western blot analysis of folate receptor $\alpha(F R \alpha)$ in A549 and KB cell lines. (B) Cell viability induced by QD-Ctrl (black columns) and QD-FA (red columns) in KB cells. KB cells were incubated in 96 well-plate for $24 \mathrm{~h}$ and treated with several QD concentrations for $24 \mathrm{~h}$. Results are expressed in percentage to control non-treated samples. spectrophotometer (Perkin Elmer, Waltham, USA), emission spectra were obtained using $340 \mathrm{~nm}$ excitation, while excitation spectra were obtained using $608 \mathrm{~nm}$ emission settings. The average number of FA per QD was determined from UV absorption spectroscopy, using a molar extinction coefficient of $25,000 \mathrm{M}^{-1} \mathrm{~cm}^{-1}$ at $283 \mathrm{~nm}$ after subtraction of the QD absorbance. All measurements were made at room temperature.

Effects of QDs on the viability of KB and A549 cells line were evaluated using the MTT assay. Briefly, cells were plated into 96-well plates and after $24 \mathrm{~h}$ the culture medium was replaced by $200 \mu \mathrm{l}$ QDs in Dulbecco's Modified Eagle Medium (DMEM) supplemented with $1 \mathrm{mM}$ sodium pyruvate (Thermo Fisher Scientific, Waltham, USA) and $10 \%$ fetal bovine serum (FBS) suspension at concentrations of 0-200 nM. Controls were realized with untreated cells (in DMEM $1 \mathrm{mM}$ pyruvate $10 \%$ FBS) taken as $100 \%$ viability. Absorbance of DQs-treated cells was normalized to the control values. After the end of incubation $(24 \mathrm{~h})$, cells were washed, $50 \mathrm{ml}$ MTT $(2 \mathrm{mg} / \mathrm{ml})$ was added to each well and incubated at $37^{\circ} \mathrm{C}$ for $2 \mathrm{~h}$. The water-insoluble formazan crystals were solubilized with dimethyl sulfoxide (DMSO) and optical density was recorded on a microplate photometer (Multiskan Ascent, Thermo Labsystems, Finland) at $540 \mathrm{~nm}$.

\subsection{Cell culture and spheroid formation}

KB oral cancer (ATTC $n^{\circ}$.CCL-17) and A549 lung carcinoma (ATTC $n^{\circ}$.CRM-CCL-185) cells were purchased from American Type Culture Collection (ATCC, Manassas, USA) and cultured according to standard mammalian tissue culture protocols and sterile technique. Both cell lines were cultured in DMEM supplemented with $1 \mathrm{mM}$ sodium pyruvate (Thermo Fisher Scientific, Waltham, USA) and 10\% FBS, using Falcon Tissue Culture Treated Flasks.

KB spheroids were obtained by using the spinner flask technique. According to preliminary studies, the application of other formation techniques such as hanging drop or liquid overlay resulted in formation of flattened disks from KB cells instead of spheres (data not shown). Briefly, $10 \mathrm{ml}$ of $2.5 \times 10^{4}$ cells suspension in DMEM supplemented with $10 \%$ FBS were incubated in $1 \%$ agarose (Sigma-Aldrich, SaintLouis, USA) coated flask $75 \mathrm{~cm}^{2}$ and incubated at $37^{\circ} \mathrm{C}, 5 \% \mathrm{CO}_{2}$, maximal hygrometry. Every $24 \mathrm{~h}, 10 \mathrm{ml}$ of completed media were added and the suspension was divided between two new agarose coated flasks. After three days of culture, whole cell suspension was placed into spinner flask (Thermo Fisher Scientific, Waltham, USA) and incubated at $37^{\circ} \mathrm{C}, 5 \% \mathrm{CO}_{2}$, maximal hygrometry under $75 \mathrm{rpm}$ agitation for seven days.

\subsection{Western blot analysis}

Cellular lysates for Western analyses were prepared in RIPA buffer (25 mM Tris, $150 \mathrm{mM} \mathrm{NaCl}, 0.1 \%$ SDS, $1 \%$ Triton X-100 ${ }^{\circ}, 0.5 \%$ sodium deoxycholate and PMSF $0.1 \mathrm{mM}$ ). Protein concentration was determined using DC Protein Assay (Biorad, Hercules, USA). Samples containing $20 \mu \mathrm{g}$ of total cellular protein were denatured $30 \mathrm{~min}$ at $37^{\circ} \mathrm{C}$ with Laemmli buffer and electrophoresed on a $10 \%$ SDS-PAGE gel and electroblotted onto a $0.2-\mu \mathrm{m}$ nitrocellulose membrane. To block nonspecific binding, the membrane was incubated at room temperature for $1 \mathrm{~h}$ with $5 \%$ nonfat dry milk in $10 \mathrm{mmol} / \mathrm{L}$ Tris-HCl buffer ( $\mathrm{pH} 8.0$ ) containing $150 \mathrm{mmol} / \mathrm{L} \mathrm{NaCl}$ and $0.05 \%$ Tween 20 (TBST). The blot was probed for $1 \mathrm{~h}$ with primary FR- $\alpha$ monoclonal antibody (Thermo Fisher Scientific, Waltham, USA) and washed three times for $15 \mathrm{~min}$ with TBST. The specific protein was detected using secondary horseradish peroxidase-conjugated immunoglobulin G. The membrane was probed with secondary antibody for $45 \mathrm{~min}$ and washed three times for 15 min, each with TBST. Proteins were visualized using Clarity ECL Western blot substrate kit (Biorad, Hercules, USA) and Azure c600 imager (Azure Biosystems, Dublin, USA).

\subsection{Epifluorescence microscopy}

KB or A549 cells were plated in Labtek Chamber at $2.5 \times 10^{4}$ cells per $\mathrm{ml}$ of DMEM, supplemented with $10 \%$ FBS. After $24 \mathrm{~h}$, the medium was discarded, and cells were washed three times with phosphate buffer saline (PBS). PBS was then replaced with FA-free or FA-saturated DMEM supplemented with 10\% FBS for $2 \mathrm{~h}$. Afterwards, QDs were added at $100 \mathrm{nM}$ for $4 \mathrm{~h}$. Before microscopy analysis, the medium was discarded, and cells were washed three times with PBS.

$\mathrm{KB}$ spheroids were treated in a similar manner, but on agarosecoated 12 well plates instead of Labtek. Spheroids were washed three time with DMEM 10\% FBS and either placed on glass slide prior to microscope imaging or frozen at $(-80){ }^{\circ} \mathrm{C}$ in optimal cutting temperature prior to cryosection. Spheroids were frozen in Tissue-Tek ${ }^{\circ}$ O.C.T. $^{\mathrm{m}}$ and $14 \mu \mathrm{m}$ thick sections were used for epifluorescence microscopy. Central section cuts with uniform round shape (around $500 \mu \mathrm{m}$ in diameter) were chosen for fluorescence profile statistics. Ten radial lines (regions of interest, ROI) were randomly drawn in the images to obtain the distribution profile of QDs. Statistics was achieved by 3 independent experiments ( 15 different spheroid cuts).

Fluorescent microscopy was performed on AX-70 Provis (Olympus, Tokyo, Japan) equipped with a $100 \mathrm{~W}$ mercury vapor lamp and a Peltier cooled CCD camera DP72 (Olympus, Tokyo, Japan). The filter was set at $400-440 \mathrm{~nm}$ band pass excitation associated with a $570 \mathrm{~nm}$ dichroic mirror and a $590 \mathrm{~nm}$ long pass emission filter for QDs fluorescence measurements. Images were converted on 8-bits format and analyzed with ImageJ software [30]. Signal-to-background-ratio (SBR) was calculated as:

$$
S B R=\frac{\text { Area of cell } \times \text { Mean fluorescence intensity of cell }}{\text { Area of cell } \times \text { Mean fluorescence intensity of background }}
$$



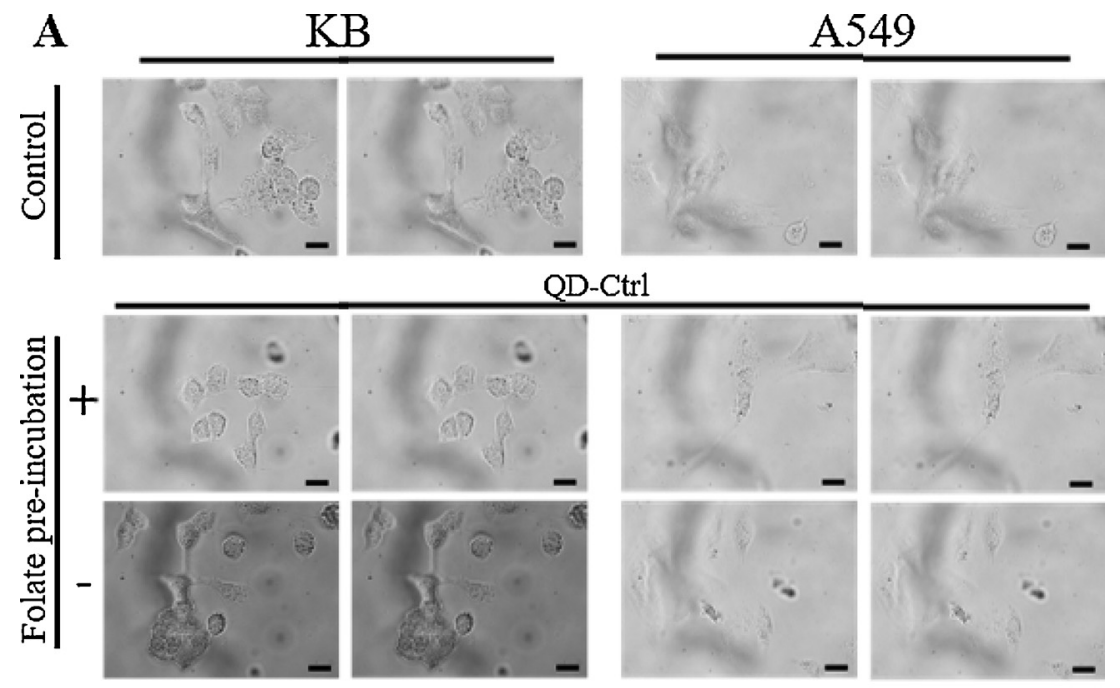

QD-FA

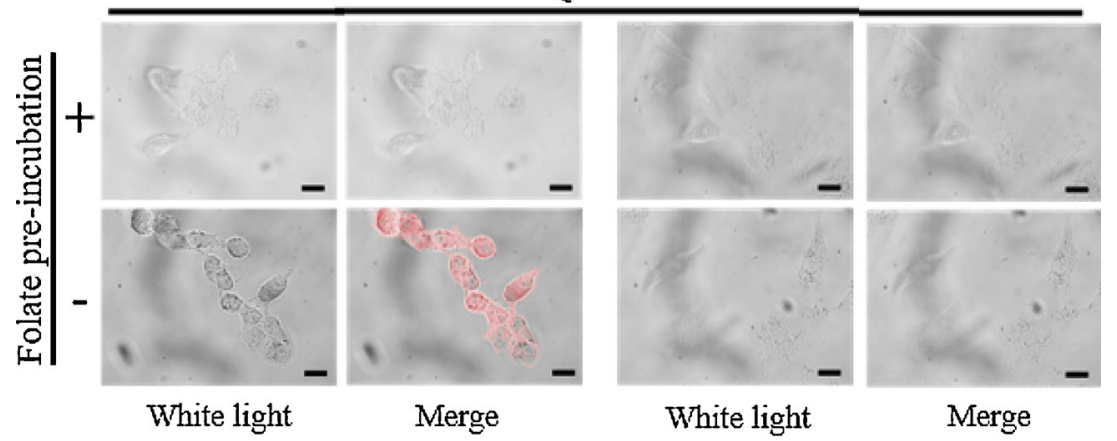

B

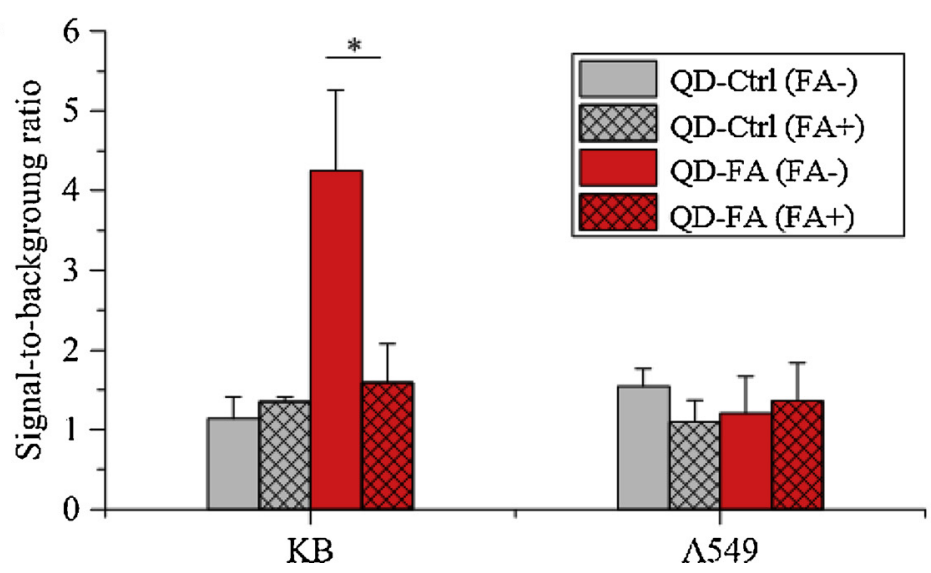

Fig. 3. (A) The images of KB and A549 monolayer cells treated with QDs for $4 \mathrm{~h}$ with or without folic acid preincubation. (B) Fluorescence signal-to-background ratio was measured for each condition. The fluorescence was excited at $405-445 \mathrm{~nm}$ and registered up to $590 \mathrm{~nm}$. The concentration of QDs was $100 \mathrm{nM}$. Scale bar $=10 \mu \mathrm{m} ; *$ : p-value $<0,05$.

\subsection{Statistical analysis}

$R$ software ( $R$ development Core team) was used for all statistical analyses. Mean value and standard deviation were calculated using descriptive statistics. $t$-Test was employed as the statistical method, $\mathrm{p}<0.05$ was considered to be statistically significant.

\section{Results and discussion}

\subsection{QD characterization}

The QDs were coated with a copolymeric ligand displaying sulfobetaine groups to ensure a good solubility in water and low nonspecific adsorption [28]. The total hydrodynamic diameter was $18 \mathrm{~nm}$ as measured in [31]. A small $(\approx 15 \%)$ portion of primary amine groups was inserted into the sulfobetaine monomers to enable conjugation with folic acid (Scheme 1). A poly(ethylene glycol) spacer was used to ensure a good availability of the terminal folic acid. Unreacted primary amines were then reacted with carboxylic acid-terminated short molecule to limit nonspecific interactions.

The QDs were characterized spectroscopically before (QD-Ctrl) and after (QD-FA) their conjugation with FA. Fig. 1 displays the spectra of absorption, fluorescence excitation and fluorescence emission of both targeted and non-targeted QDs in buffer solution. The absorption spectra are typical for QDs [32], showing a gradual decrease in extinction with increase in the wavelength. A comparative analysis 

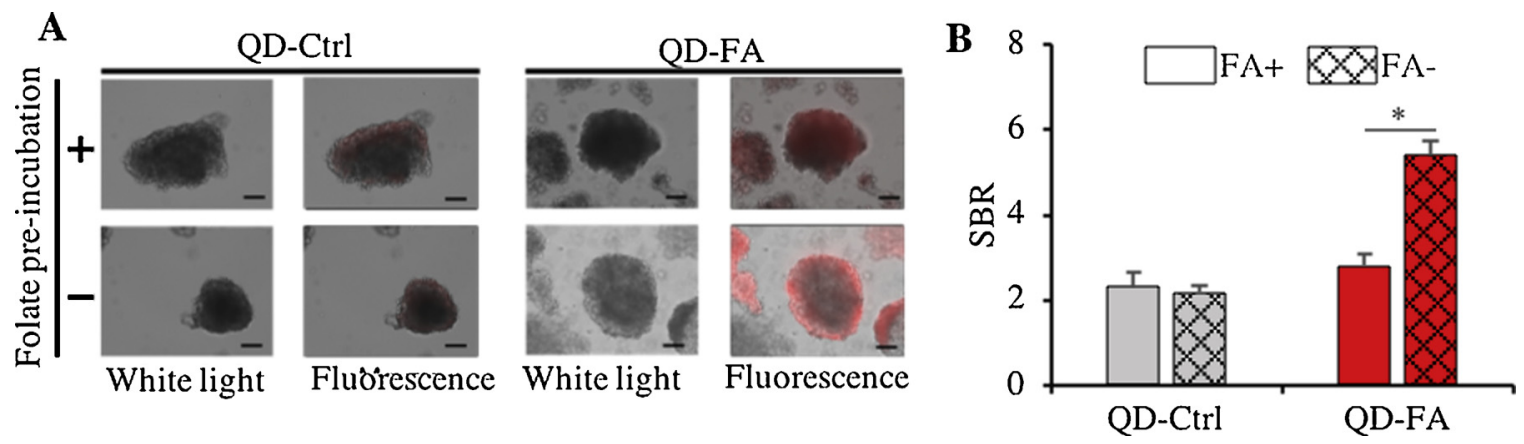

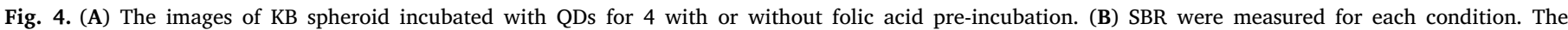
fluorescence was excited at $\lambda=405-445 \mathrm{~nm}$ and registered above $590 \mathrm{~nm}$. The QD concentration was $100 \mathrm{nM}$. Scale bar $=100 \mu \mathrm{m}$; $*$ p-value $<0,05$.

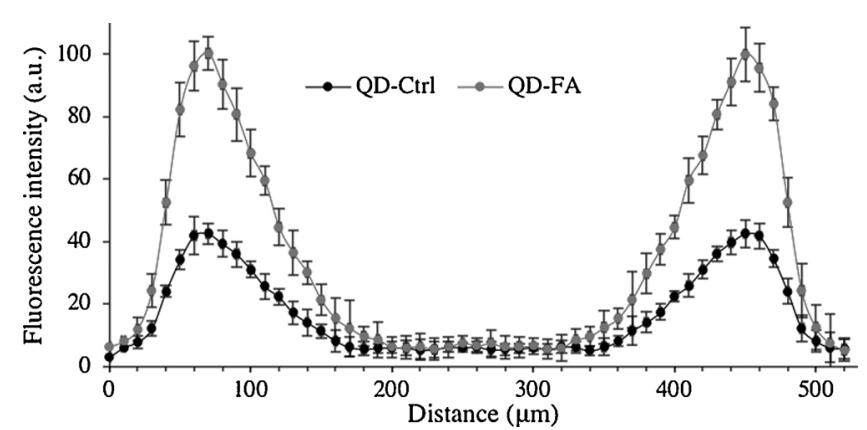

Fig. 5. Mean fluorescence distribution profiles of QD-Ctrl and QD-FA across the cut of KB spheroid after $4 \mathrm{~h}$ incubation with QDs.

demonstrated that conjugation of QDs with FA did not significantly affect their absorbance characteristics in the range of $325-650 \mathrm{~nm}$. The study of absorbance of purified QD-FA in UV-C range $(250-300 \mathrm{~nm})$ demonstrated the presence of FA characteristic bands, corresponding to approximately 10-15 FA molecules bound per QD (data not shown).

More dramatic changes appear in the fluorescence spectra of QDCtrl upon their conjugation with FA. The conjugation results in significant quenching of fluorescence. The QD fluorescence quantum yield drops from ca. 0.50 before FA conjugation to ca. 0.25 after conjugation with FA. FA-induced quenching of QDs is a commonly reported albeit not perfectly understood issue [33]. Fluorescence quenching in such systems is proportional to the ratio between FA and QD and could be used for FA quantification [22]. At the same time, the shape of spectra remains unchanged, no shift was observed, thus indicating that the QD materials remained intact upon folate conjugation.

\subsection{The interaction of $Q D s$ with $2 D$ monolayer tumor cells}

The in vitro studies were conducted in two tumor cell lines, $\mathrm{KB}$ and A549. KB oral squamous cell carcinoma cells are considered as FR- $\alpha$ positive cell line. As a negative control we used human lung carcinoma A549 cells which are folate receptor negative [34]. To confirm it, Western blot analysis was performed to assess FR expression in both cell lines (Fig. 2A). As follows from Fig. 2A, KB cells displayed strong expression of FRa, while A549 were FR $\alpha$-free.

We further assessed the QDs-induced toxicity in function of QDs concentrations in both cell lines using MTT assay. Even at the highest QDs concentration $(200 \mathrm{nM})$, both types of QDs did not cause significant changes in metabolic activity neither in KB cells (Fig. 2B) nor in A549 cells (data not shown).

The accumulation of QDs in monolayer cells was studied using epifluorescence microscopy. Fig. 3 exhibits the images of KB and A549 cells treated with QD-Ctrl or QD-FA for $4 \mathrm{~h}$. To analyze the specificity of targeting, we conducted the experiments under two conditions: with and without pre-incubation of cells with FA.
Fluorescence from QD-FA was observed only in FA-starved KB cells, while fluorescence was undetectable in A459 cells or FA pre-incubated $\mathrm{KB}$ cells, as shown in Fig. 3A. The quantitative analysis of fluorescence images is presented in Fig. 3B. According to obtained data, the saturation of folate receptors results in a significant inhibition of QD-FA uptake $(\mathrm{SBR}=1.6 \pm 0.5 v s \mathrm{SBR}=4.2 \pm 1.2$ without FA pre-incubation). Likewise, QDs accumulation was not detectable in the case of folate receptor-negative A549 cells, with or without FA pre-incubation $(\mathrm{SBR} \approx 1)$. These observations confirm the FR- $\alpha$ receptor specific endocytosis of QD-FA in monolayer cells.

\subsection{The distribution of QDs in MCTSs}

The experiments in 2D cells confirmed the essential role of folate in the intracellular QD targeting. However, 2D model does not fully reflect the interactions of QDs with tumors. To this purpose, we developed a FR- $\alpha$ receptor positive tumors spheroid KB cell model. This 3D model better reflects tumors characteristics, such as heterogeneous cellular growth with peripheral proliferating cells, a quiescent core, several gradients, decreasing from the periphery to the center of the spheroid such as oxygen, $\mathrm{pH}$ and nutrients and a presence of extracellular matrix. Thus, spheroids are useful models to study nanoparticles penetration and their potential theranostic applications [35].

Uptake of QD-Ctrl and QD-FA in KB spheroids was characterized by a significantly higher uptake of QD-FA after folate starvation $(\mathrm{SBR}=5.4 \pm 0.8)$, while FA pre-incubated spheroids demonstrated low fluorescence signal after incubation $(S B R=2.8 \pm 0.2)$ (Fig. 4). Thus, similar to 2D cells, specificity of QD-FA was also confirmed in FR$\alpha$ receptor positive 3D tumor spheroid model. It is interesting to note that QD-Ctrl displayed low but still detectable fluorescence signal irrespective of pre-incubation conditions in spheroid model $(\mathrm{SBR}=2.4$ \pm 0.4 or SBR $=2.2 \pm 0.2$ with or without FA pre-incubation respectively). This low fluorescence signal could probably correspond to QDs localization in extracellular space.

To study QD-FA and QD-Ctrl distribution in KB spheroids, MTCSs were cut and imaged by epifluorescence microscopy. Fig. 5 demonstrates the QDs distribution as a function of depth into the spheroid. Both QDs were mainly localized in the outer layer of FA-starved spheroids with rapidly decreasing fluorescence from the periphery to the center of the spheroid. Penetration profiles were similar for both QDs with penetration depth never exceeding $100 \mu \mathrm{m}$. We hypothesized that QD penetration could be limited by the lack of adenosine triphosphate (ATP) in the quiescent cells which are close to the spheroid core [36]. By this way, the deficit of ATP resulting in a lower probability to absorb QD by FR- $\alpha$-mediated endocytosis [13].

\section{Conclusions}

We have demonstrated the effectiveness of FA conjugated QDs to target tumors spheroid model thus confirming the crucial role of FR $\alpha$ 
receptor as a target. Compared with untargeted QDs, QD-FA has few folic acids added on their surface, otherwise both types of QDs share similar charge and protein corona and therefore there are expected to display similar spheroid penetration. Thus, higher SBR obtained in KB spheroids treated with QD-FA is clearly due to improved uptake and is not related to the different penetration abilities of QDs.

Further improvement of QD-FA targeting ability could be performed using dual targeting with different targeting agents, such as FA and cyclic RGD [37]. In vivo experiment with FA targeted nanoparticles have also demonstrated the critical importance of a high load of FA per NPs [38], however we should consider a high probability of QDs fluorescence quenching by FA [39]. Therefore, further optimization of QD-FA conjugates is warranted and spheroids model here could be of big value.

\section{Conflicts of interest}

The authors declare no conflict of interest.

\section{Funding}

This research was funded by the Ligue Nationale Contre le Cancer, the Institut de Cancérologie de Lorraine and the Lorraine Region.

\section{Acknowledgment}

The authors thank Dominique Marius Le Prince (Nancy, France) for the proof-reading and corrections of the manuscript.

\section{References}

[1] Global Burden of Disease Cancer Collaboration, C. Fitzmaurice, C. Allen, R.M. Barber, L. Barregard, Z.A. Bhutta, et al., Global, regional, and national cancer incidence, mortality, years of life lost, years lived with disability, and disabilityadjusted life-years for 32 cancer groups, 1990 to 2015: a systematic analysis for the global burden of disease study, JAMA Oncol. 3 (2017) 524-548, https://doi.org/10. 1001/jamaoncol.2016.5688.

[2] S. Swain, P.K. Sahu, S. Beg, S.M. Babu, Nanoparticles for cancer targeting: current and future directions, Curr. Drug Deliv. 13 (2016) 1290-1302.

[3] C.T. Matea, T. Mocan, F. Tabaran, T. Pop, O. Mosteanu, C. Puia, C. Iancu, L. Mocan, Quantum dots in imaging, drug delivery and sensor applications, Int. J. Nanomed. 12 (2017) 5421-5431, https://doi.org/10.2147/IJN.S138624.

[4] A. Robe, E. Pic, H.-P. Lassalle, L. Bezdetnaya, F. Guillemin, F. Marchal, Quantum dots in axillary lymph node mapping: biodistribution study in healthy mice, BMC Cancer 8 (2008) 111, https://doi.org/10.1186/1471-2407-8-111.

[5] M. Fang, M. Chen, L. Liu, Y. Li, Applications of quantum dots in cancer detection and diagnosis: a review, J. Biomed. Nanotechnol. 13 (2017) 1-16.

[6] K. Gonda, M. Miyashita, H. Higuchi, H. Tada, T.M. Watanabe, M. Watanabe, T. Ishida, N. Ohuchi, Predictive diagnosis of the risk of breast cancer recurrence after surgery by single-particle quantum dot imaging, Sci. Rep. 5 (2015) 14322, https://doi.org/10.1038/srep14322.

[7] D. Radenkovic, H. Kobayashi, E. Remsey-Semmelweis, A.M. Seifalian, Quantum dot nanoparticle for optimization of breast cancer diagnostics and therapy in a clinical setting, Nanomedicine Nanotechnol. Biol. Med. 12 (2016) 1581-1592, https://doi. org/10.1016/j.nano.2016.02.014.

[8] T. Mangeolle, I. Yakavets, S. Marchal, M. Debayle, T. Pons, L. Bezdetnaya, F. Marchal, T. Mangeolle, I. Yakavets, S. Marchal, M. Debayle, T. Pons, L. Bezdetnaya, F. Marchal, Fluorescent nanoparticles for the guided surgery of ovarian peritoneal carcinomatosis, Nanomaterials 8 (2018) 572, https://doi.org/ 10.3390/nano8080572.

[9] S. Kamila, C. McEwan, D. Costley, J. Atchison, Y. Sheng, G.R.C. Hamilton, C. Fowley, J.F. Callan, Diagnostic and therapeutic applications of quantum dots in nanomedicine, Top. Curr. Chem. 370 (2016) 203-224, https://doi.org/10.1007/ 978-3-319-22942-3 7

[10] T. Deng, J. Wang, Y. Li, Z. Han, Y. Peng, J. Zhang, Z. Gao, Y. Gu, D. Deng, Quantum dots-based multifunctional nano-prodrug fabricated by ingenious self-assembly strategies for tumor theranostic, ACS Appl. Mater. Interfaces 10 (2018) 27657-27668, https://doi.org/10.1021/acsami.8b08512.

[11] J.V. Jokerst, T. Lobovkina, R.N. Zare, S.S. Gambhir, Nanoparticle PEGylation for imaging and therapy, Nanomedicine 6 (2011) 715-728, https://doi.org/10.2217 nnm.11.19.

[12] M. Fernández, F. Javaid, V. Chudasama, Advances in targeting the folate receptor in the treatment/imaging of cancers, Chem. Sci. 9 (2018) 790-810, https://doi.org/ 10.1039/C7SC04004K.

[13] A. Cheung, H.J. Bax, D.H. Josephs, K.M. Ilieva, G. Pellizzari, J. Opzoomer, J. Bloomfield, M. Fittall, A. Grigoriadis, M. Figini, S. Canevari, J.F. Spicer,
A.N. Tutt, S.N. Karagiannis, Targeting folate receptor alpha for cancer treatment, Oncotarget 7 (2016) 52553-52574, https://doi.org/10.18632/oncotarget.9651.

[14] G.L. Zwicke, G.A. Mansoori, C.J. Jeffery, Utilizing the folate receptor for active targeting of cancer nanotherapeutics, Nano Rev. 3 (2012), https://doi.org/10. 3402/nano.v3i0.18496.

[15] R.J. Lutz, Targeting the folate receptor for the treatment of ovarian cancer, Transl. Cancer Res. 4 (2015) 118-126, https://doi.org/10.21037/3838.

[16] D.J. O'Shannessy, E.B. Somers, J. Maltzman, R. Smale, Y.-S. Fu, Folate receptor alpha (FRA) expression in breast cancer: identification of a new molecular subtype and association with triple negative disease, SpringerPlus 1 (2012), https://doi.org/ 10.1186/2193-1801-1-22.

[17] D.J. O'Shannessy, G. Yu, R. Smale, Y.-S. Fu, S. Singhal, R.P. Thiel, E.B. Somers, A. Vachani, Folate receptor alpha expression in lung cancer: diagnostic and prognostic significance, Oncotarget 3 (2012) 414-425, https://doi.org/10.18632/ oncotarget. 489.

[18] L. Xu, Q. Bai, X. Zhang, H. Yang, Folate-mediated chemotherapy and diagnostics: an updated review and outlook, J. Control. Release 252 (2017) 73-82, https://doi.org/ 10.1016/j.jconrel.2017.02.023.

[19] I.B. Bwatanglang, F. Mohammad, N.A. Yusof, J. Abdullah, M.Z. Hussein, N.B. Alitheen, N. Abu, Folic acid targeted Mn:ZnS quantum dots for theranostic applications of cancer cell imaging and therapy, Int. J. Nanomed. 11 (2016) 413-428, https://doi.org/10.2147/IJN.S90198.

[20] I.B. Bwatanglang, F. Mohammad, N.A. Yusof, J. Abdullah, N.B. Alitheen, M.Z. Hussein, N. Abu, N.E. Mohammed, N. Nordin, N.R. Zamberi, S.K. Yeap, In vivo tumor targeting and anti-tumor effects of 5-fluororacil loaded, folic acid targeted quantum dot system, J. Colloid Interface Sci. 480 (2016) 146-158, https://doi.org/ 10.1016/j.jcis.2016.07.011.

[21] R.-Y. Zhang, Z.-Y. Wang, X.-Q. Yang, Y. Xuan, K. Cheng, C. Li, X.-L. Song, Jie An, X.L. Hou, Y.-D. Zhao, Folic acid modified Pluronic F127 coating Ag2S quantum dot for photoacoustic imaging of tumor cell-targeting, Nanotechnology 29 (2018) 055101, https://doi.org/10.1088/1361-6528/aa9acc.

[22] Folic acid-conjugated core/shell $\mathrm{ZnS}: \mathrm{Mn} / \mathrm{ZnS}$ quantum dots as targeted probes for two photon fluorescence imaging of cancer cells, Acta Biomater. 7 (2011) 1327-1338, https://doi.org/10.1016/j.actbio.2010.10.012.

[23] V. Morosini, T. Bastogne, C. Frochot, R. Schneider, A. François, F. Guillemin, M. Barberi-Heyob, Quantum dot-folic acid conjugates as potential photosensitizers in photodynamic therapy of cancer, Photochem. Photobiol. Sci. 10 (2011) 842-851, https://doi.org/10.1039/c0pp00380h.

[24] R. Edmondson, J.J. Broglie, A.F. Adcock, L. Yang, Three-dimensional cell culture systems and their applications in drug discovery and cell-based biosensors, Assay Drug Dev. Technol. 12 (2014) 207-218, https://doi.org/10.1089/adt.2014.573.

[25] I. Yakavets, I. Yankovsky, M. Millard, L. Lamy, H.-P. Lassalle, A. Wiehe, V. Zorin, L. Bezdetnaya, The alteration of temoporfin distribution in multicellular tumor spheroids by $\beta$-cyclodextrins, Int. J. Pharm. 529 (2017) 568-575, https://doi.org/ 10.1016/j.ijpharm.2017.07.037.

[26] Y.A. Yang, H. Wu, K.R. Williams, Y.C. Cao, Synthesis of CdSe and CdTe nanocrystals without precursor injection, Angew. Chem. Int. Ed. 44 (2005) 6712-6715, https:// doi.org/10.1002/anie.200502279.

[27] R. Xie, U. Kolb, J. Li, T. Basché, A. Mews, Synthesis and characterization of highly luminescent CdSe - core CdS/Zn0.5Cd0.5S/ZnS multishell nanocrystals, J. Am. Chem. Soc. 127 (2005) 7480-7488, https://doi.org/10.1021/ja042939g.

[28] M. Tasso, E. Giovanelli, D. Zala, S. Bouccara, A. Fragola, M. Hanafi, Z. Lenkei, T. Pons, N. Lequeux, Sulfobetaine-vinylimidazole block copolymers: a robust quantum dot surface chemistry expanding bioimaging's horizons, ACS Nano 9 (2015) 11479-11489, https://doi.org/10.1021/acsnano.5b05705.

[29] C.A. Leatherdale, W.-K. Woo, F.V. Mikulec, M.G. Bawendi, On the absorption cross section of CdSe nanocrystal quantum dots, J. Phys. Chem. B 106 (2002) 7619-7622, https://doi.org/10.1021/jp025698c.

[30] C.T. Rueden, J. Schindelin, M.C. Hiner, B.E. DeZonia, A.E. Walter, E.T. Arena, K.W. Eliceiri, ImageJ2: ImageJ for the next generation of scientific image data, BMC Bioinform. 18 (2017) 529, https://doi.org/10.1186/s12859-017-1934-z.

[31] L. Trapiella-Alfonso, T. Pons, N. Lequeux, L. Leleu, J. Grimaldi, M. Tasso, E. Oujagir, J. Seguin, F. d'Orlyé, C. Girard, B.-T. Doan, A. Varenne, Clickable-Zwitterionic copolymer capped-quantum dots for in vivo fluorescence tumor imaging, ACS Appl. Mater. Interfaces 10 (2018) 17107-17116, https://doi.org/10.1021/acsami. 8b04708.

[32] M. Tasso, M.K. Singh, E. Giovanelli, A. Fragola, V. Loriette, M. Regairaz, F. Dautry, F. Treussart, Z. Lenkei, N. Lequeux, T. Pons, Oriented bioconjugation of unmodified antibodies to quantum dots capped with copolymeric ligands as versatile cellular imaging tools, ACS Appl. Mater. Interfaces 7 (2015) 26904-26913, https://doi.org/ 10.1021/acsami.5b09777.

[33] P. Suriamoorthy, X. Zhang, G. Hao, A.G. Joly, S. Singh, M. Hossu, X. Sun, W. Chen, Folic acid-CdTe quantum dot conjugates and their applications for cancer cell targeting, Cancer Nanotechnol. 1 (2010) 19-28, https://doi.org/10.1007/s12645-0100003-3.

[34] J.A. Reddy, L.S. Haneline, E.F. Srour, A.C. Antony, D.W. Clapp, P.S. Low, Expression and functional characterization of the beta-isoform of the folate receptor on CD34(+) cells, Blood 93 (1999) 3940-3948.

[35] M. Millard, I. Yakavets, V. Zorin, A. Kulmukhamedova, S. Marchal, L. Bezdetnaya, Drug delivery to solid tumors: the predictive value of the multicellular tumor spheroid model for nanomedicine screening, Int. J. Nanomed. 12 (2017) 7993-8007, https://doi.org/10.2147/IJN.S146927.

[36] F. Hirschhaeuser, H. Menne, C. Dittfeld, J. West, W. Mueller-Klieser, L.A. KunzSchughart, Multicellular tumor spheroids: an underestimated tool is catching up again, J. Biotechnol. 148 (2010) 3-15, https://doi.org/10.1016/j.jbiotec.2010.01. 012. 
[37] C. Jang, J.H. Lee, A. Sahu, G. Tae, The synergistic effect of folate and RGD dual ligand of nanographene oxide on tumor targeting and photothermal therapy in vivo, Nanoscale 7 (2015) 18584-18594, https://doi.org/10.1039/c5nr05067g.

[38] J.H. Lee, A. Sahu, C. Jang, G. Tae, The effect of ligand density on in vivo tumor targeting of nanographene oxide, J. Control. Release 209 (2015) 219-228, https:/ doi.org/10.1016/j.jconrel.2015.04.035.

[39] Copper- or manganese-doped ZnS quantum dots as fluorescent probes for detecting folic acid in aqueous media, J. Lumin. 132 (2012) 987-991, https://doi.org/10. 1016/j.jlumin.2011.12.014. 